\title{
Semantic Processing and Orthographic Specificity in Hemispatial Neglect
}

\section{Citation}

McGlinchey-Berroth, Regina, William P. Milberg, Mieke Verfaellie, Laura Grande, Mark D'Esposito, and Michael Alexander. 1996. "Semantic Processing and Orthographic Specificity in Hemispatial Neglect." Journal of Cognitive Neuroscience 8 (3): 291-304. doi:10.1162/ jocn.1996.8.3.291.

\section{Permanent link}

http://nrs.harvard.edu/urn-3:HUL.InstRepos:41542969

\section{Terms of Use}

This article was downloaded from Harvard University's DASH repository, and is made available under the terms and conditions applicable to Other Posted Material, as set forth at http:// nrs.harvard.edu/urn-3:HUL.InstRepos:dash.current.terms-of-use\#LAA

\section{Share Your Story}

The Harvard community has made this article openly available. Please share how this access benefits you. Submit a story.

Accessibility 


\title{
Semantic Processing and Orthographic Specificity in Hemispatial Neglect
}

\author{
Regina McGlinchey-Berroth and William P. Milberg \\ Department of Veterans Affairs Medical Center, West Roxbury and \\ Harvard University Medical School \\ Mieke Verfaellie \\ Boston University School of Medicine \\ Laura Grande \\ Department of Veterans Affairs Medical Center, West Roxbury
}

\author{
Mark D'Esposito \\ Hospital of the University of Pennsylvania
}

Michael Alexander

Boston University School of Medicine

\begin{abstract}
Two sets of experiments, each consisting of a semantic priming task and a discrimination task, investigated the proceedings of lexical information in the neglected visual field. In the semantic priming task, subjects made lexical decisions to target words preceded by lateralized word primes; in the discrimination task, they indicated which of two words corresponded to a target word presented to the left visual field (LVF) or right visual field (RVF). The first set of experiments indicated that although patients were unable to discriminate words presented in the LVF, they showed significant priming when LVF primes were followed by semantically related targets compared to unrelated targets. The second set of experiments further examined the nature of this priming effect by comparing priming in a condition in which primes were semantically
\end{abstract}

\section{INTRODUCTION}

An increasing body of evidence suggests that patients with hemispatial neglect following right hemisphere lesions process visual information present in the left visual field that they cannot respond to directly. Perhaps the earliest indication that patients with neglect had at least some access to information present in the affected field was described by Kinsbourne and Warrington (1962), who noted that the reading errors committed by patients with right hemisphere lesions maintain the length of the words presented to them in a tachistoscopic reading task. Volpe, Ledoux, and Gazzaniga (1979) found that patients who could not name pictures of objects presented in the neglected field performed above chance on a cross-field matching task. Marshall and Hal- related to the target word (e.g., TEA-CUP) and a condition in which primes were unrelated to the target word, but orthographically similar to a related prime (e.g., PEA-CUP). This experiment replicated the previously established semantic priming effects and demonstrated significant negative priming for targets preceded by LVF primes that were orthographically similar to a semantically related word. Again, patients performed at chance in the forced-choice discrimination task when targets were presented in the LVF. These findings indicate that semantic processing of neglected lexical information is based on fully specified perceptual and orthographic information. A lateral inhibitory mechanism is proposed that maximizes the probability, albeit unsuccessfully, that neglected orthographic information will reach awareness.

ligan (1988) showed that a patient's preference for a picture of a house was influenced by the presence or absence of "flames" on the left side of the picture even though she judged both pictures to be identical.

More recently, semantic processing of neglected visual information was reported in a study by McGlinchey-Berroth, Milberg, Verfaellie, Alexander, and Kilduff (1993). In that study, implicit processing was assessed within the context of a semantic priming, lexical decision task in which patients judged the lexical status (i.e., word/nonword) of target letter strings that were presented in midline and preceded by lateralized picture primes. On critical trials, the priming displays were composed of one line drawing that was either related or unrelated to the target word and one nonsense figure. Like normal control subjects, neglect patients showed equivalent 


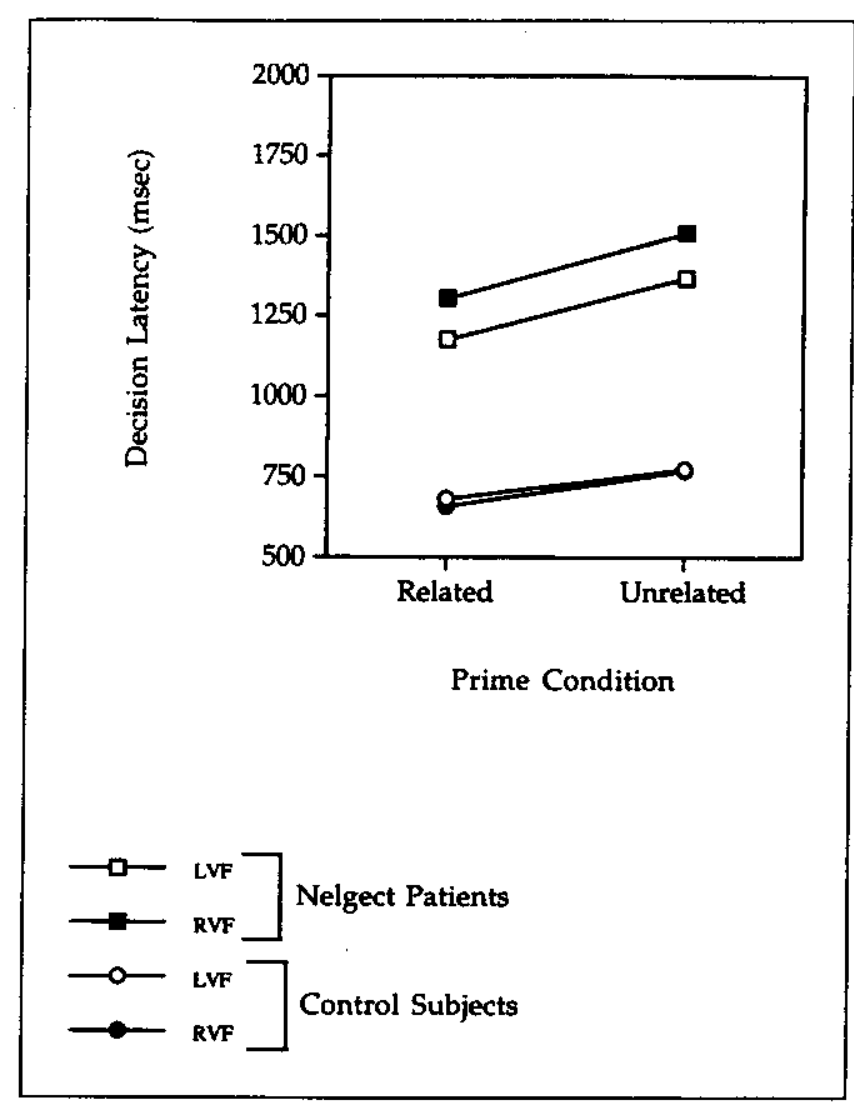

Figure 1. Mean lexical decision latencies as a function of priming condition and visual field in Experiment la for neglect patients and normal control subjects.

\section{Experiment 1a: Semantic Priming from Lexical Information}

\section{Accuracy}

Due to the high level of performance in this task, statistical analysis of the accuracy data was not possible. In total, there were only two errors in the critical conditions for the patients; both were made by the same patient (patient 3): one following an LVF unrelated prime and one following an RVF unrelated prime. Similarly for normal subjects; there were onty 3 errors in total made by 3 different subjects: 2 occurred following LVF unrelated primes and one following an RVF unrelated prime.

\section{Decision Latency}

As the result of generalized attentional deficits that are common in hemispatial neglect (Mesulam, 1985), patients occasionally lost set during the task. For trials in which this occurred (defined individually as decision latencies $\geq 2$ standard deviations above the mean for the condition in which the loss of set occurred), the patients were prompted to respond by guessing and that trial was removed from the data set. This procedure resulted in the loss of 3 trials for 2 of the neglect patients, 2 trials for 3 patients, and 1 trial for one other patient.
Decision latencies were subjected to a $2 \times(2 \times 2)$ repeated measures analysis of variance (ANOVA) examining the effects of the Visual Field of the priming stimulus (left versus right) and its Relatedness to the target word (related versus unrelated) across the two groups of subjects (neglect versus control). Table 1 presents the means, standard deviations, and error rates for individual neglect patients and for the normal control group. The means, standard deviations, and error rates for nonword targets and fillers are presented in Table 2.

Overall, neglect patients took $1301 \mathrm{msec}$ to judge the lexical status of targets, whereas the control subjects made these decisions in $722 \mathrm{msec}[F(1,15)=12.17$, $p<0.01]$. Neither the main effect of Visual Field $[F(1,15)=0.37]$ nor its interaction with Group $[F(1,15)=0.02]$ was significant. The relationship between the prime and target was highly significant $[F(1,15)=38.70, p<0.001]$. On average, related targets were responded to in $905 \mathrm{msec}$, whereas unrelated targets were responded to in $1016 \mathrm{msec}$. This produced an overall priming effect of $111 \mathrm{msec}$. This priming effect remained stable across Groups $[F(1,15)=2.07]$ and Visual Fields $[F(1,15)=1.17]$. Lastly, the pattern of priming for each subject group was equivalent in the left and right visual field $[F(1,15)=0.78]$, as depicted in Figure 1.

\section{Experiment 1b: Forced Choice Discrimination}

The discrimination data were analyzed in two ways. First, each subject's percentage correct was determined as a function of the visual field in which the target initially appeared. These data were then used to determine whether patients performed this task better than chance in each visual field (chance determined to lie between 32 and $68 \%$ ). Additionally, a repeated measures ANOVA was conducted examining the between subjects effect of Group and the within subjects effect of Visual Field to determine whether the pattern of performance across the visual fields was similar in the two groups of subjects.

Table 1 displays the mean percentage correct for individual neglect patients and for the control group. Clearly, neglect patients were unable to decide which word had just been presented to them in the LVF beyond what would be expected by chance. In contrast, they were able to discriminate the target word reliably when it was initially presented in the RVF. Normal control subjects performed at ceiling.

Results from the ANOVA revealed that neglect patients' overall performance of $72 \%$ correct was impaired relative to the control subjects' performance of $94 \%$ correct $[F(1,15)=13.81, p<0.01]$. Overall, RVF targets were discriminated more accurately ( $92 \%$ correct) than LVF targets ( $78 \%$ correct). However, a significant interaction between Group and Visual Field $[F(1,15)=25.60$, $p<0.001]$ indicated that this was the case only for 
Table 2. Correct Mean Response Time, Standard Deviation, and Total Errors for Experiments la and 2a: Semantic Priming in the Neglected Visual Field for Fillers and Nonword Targets

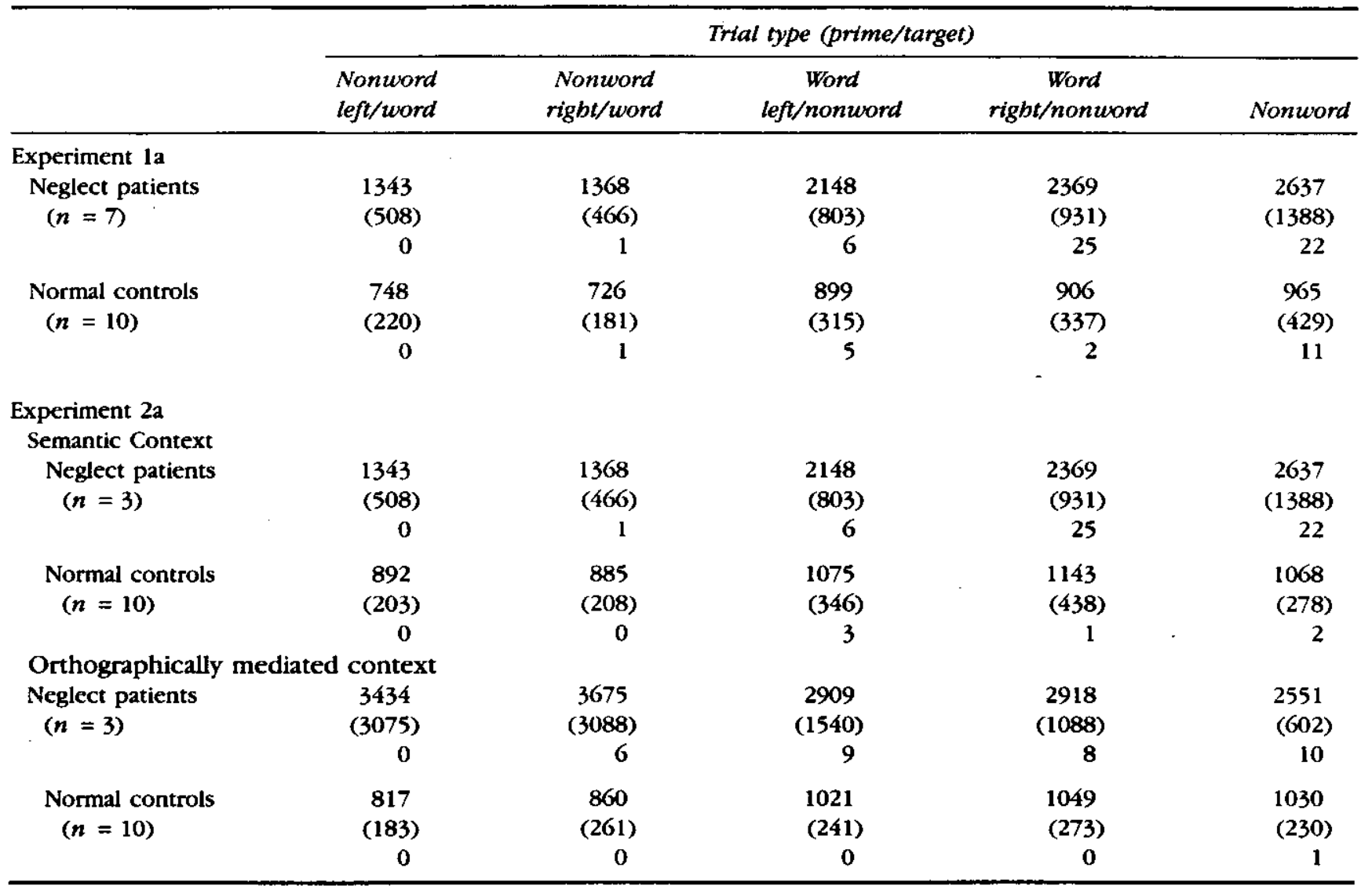

to determine the word's identity using a combination of automatic and controlled lexical access processes (see Neely, 1991). For example, suppose that when presented with "CAT" in the neglected visual field, patients actually perceive "AT." It is possible that this partially perceived word segment automatically activates a cohort of representations consistent with the perceived segment's orthographic attributes (i.e., "HAT," "CAT," "ATE," "STATE," etc.). The patient could then use this cohort to predict or guess the identity of the prime word. One problem with this view is that the orthographic cohort activated based on the perception of "AT" is sufficiently large to make the accurate prediction of "CAT" highly improbable. This position could be maintained, however, by allowing the additional possibility that activation of orthographic representations is restricted to only those lexical representations that match the input in length and letter position. This possibility is supported by studies of neglect dyslexia suggesting that the deficit occurs at a "string" level of representation (Riddoch, Hum. phreys, Cleton, \& Fery, 1990). At this level of representation, letters are thought to be coded in reference to their spatial position within letter strings, and not in reference to their absolute position in retinotopic space. Thus, word length information may be computed cor- rectly and could be used to constrain lexical access, despite the inability to specify the letter(s) present in the leftmost positions within the string. In our example, if patients are aware that the letters " $\mathrm{AT}^{n}$ are the last two letters of the prime word and are preceded by one unperceived letter, the cohort activated by "AT" would be reduced to only those representations that are three letters in length and that end in "AT." This additional constraint might make a guessing strategy more tenable.

One argument against this account is the fact that neglect patients' performance in the discrimination task was at chance in the LVF. If partial perception is sufficient to account for semantic priming, then one would also expect above chance discrimination, as partial information should also be sufficient to discriminate a LVF target from a visually unrelated foil. This argument rests, however, on the assumption that the attentional demands of the priming and discrimination task are identical. It is possible that the attentional requirements of the two tasks are sufficiently different so that partial information is available in one and not the other (see Verfaellie et al., 1995). For example, the requirement that subjects explicitly attend to information in the initial display of the discrimination task and subsequently make a judgment about that information may enhance 
two ways. The first set of analyses was concerned with the between-subjects effect of Group (neglect versus control) across the within-subjects effects of Visual Field (left versus right) and Prime Type (related versus unrelated) for each Context condition (orthographic versus semantic) separately. The second set of analyses was concerned with the effects of Context, Visual Field, and Prime Type for neglect patients and control subjects separately. While somewhat repetitive, we felt that this approach would overcome the limited amount of statistical power in the neglect group, and still provide all theoretically important comparisons.

\section{Experiment 2a: Semantic and Orthographic Priming from Lexical Information}

\section{Accuracy}

Both the patients and the control subjects performed flawlessly in the semantic condition. The same was true in the orthographic condition for the controls and two of the neglect patients. One patient, however, did have difficulty performing lexical decisions in the orthographically mediated condition, making 16 errors (40\%) in the critical conditions. Six of these errors occurred for targets following LVF primes (3 errors each in the orthographically similar and dissimilar condition), and 10 errors occurred for targets following RVF primes (5 errors each in the orthographically similar and dissimilar condition). This pattern of error data is most likely indicative of fatigue during this testing session, and not due to a qualitative performance difference in the two context conditions. Due to the overall high level of performance, statistical analysis was not conducted on the accuracy data.

\section{Decision Latency}

Trials on which patients responded $\geq 2$ standard deviations from their individual mean for that condition were excluded from the analysis. In total this procedure resulted in a loss of 9 trials. The trials in which this occurred were distributed relatively evenly across conditions and were not systematically related to any of the factors of interest. Table 3 presents the means, standard deviations, and error rates for individual neglect patients and for the normal control group. The means, standard deviations, and error rates for nonword targets and fillers are presented in Table 2.

In the semantic condition, a main effect of Group was revealed $[F(1,11)=18.40, p<0.01]$, indicating that neglect patients' decision latencies were significantly longer ( $2248 \mathrm{msec}$ ) than those of normal control subjects $(890 \mathrm{msec})$. A main effect of Prime Type $[F(1,11)=$ $21.50, p<0.001]$ also revealed that decision latencies to related targets (1155 msec) were significantly faster than to unrelated targets $(1251 \mathrm{msec})$. This indicates an overall priming effect of $96 \mathrm{msec}$. No other effects or interactions were significant.

In the orthographically mediated condition, neglect patients (2743 msec) also responded more slowly $[F(1,11)=26.94, p<0.001]$ than did normal control subjects $(877 \mathrm{msec})$. The interactions of Visual Field and Group $[F(1,11)=8.56, p<0.05]$ and Visual Field and Prime Type $[F(1,11)=6.02, p<0.05]$ were significant but were qualified by a marginally significant three-way interaction between Group, Visual Field, and Prime Type $[F(1,11)=4.07, p<0.07]$, indicating a different pattern of performance across the two subject groups. In particular, analysis of the neglect patients' data revealed a significant interaction between Prime Type and Visual Field $[F(1,2)=20.9, p<0.05]$. This interaction reflected the fact that neglect patients were significantly slower $[F(1,2)=25.51, p<0.05]$ in responding to orthographically mediated targets compared to dissimilar targets in the LVF (i.e., negative priming), whereas there was no significant effect of prime type in the RVF. Analysis of the normal control subjects' data showed a nonsignificant priming effect for orthographically mediated targets regardless of the visual field in which the prime was presented. The triple interaction between Group, Visual Field, and Prime Type, while marginal statistically, is consistent with the notion that orthographic processing in the neglected visual field differs qualitatively from that in the intact field and from that observed in normal control subjects.

Three additional ANOVAs were conducted to examine the effects of Context (orthographic versus semantic) and Visual Field within each group separately. For normal subjects, decision latencies were subjected to a $2 \times 2 \times$ 2 repeated measures ANOVA that examined the effects of Context condition (semantic versus orthographically mediated), Visual Field of the prime (left versus right), and Prime Type (related versus unrelated).

For normal subjects, decision latencies to related targets $(869 \mathrm{msec})$ were significantly faster than to unrelated targets [898 msec; $F(1,9)=6.81, p<0.05$ ]. As presented in Figure 2, a significant Context by Prime Type interaction $[F(1,9)=5.36, p<0.05]$ indicated that this priming effect was significant only given a semantically related context $[F(1,9)=5.36, p<0.05]$ and not given an orthographically similar context $[F(1,9)<1)$. No other main effects or interactions reached significance.

For neglect patients, this analysis revealed only a significant interaction of Context by Prime Type $[F(1,2)=$ $30.77, p<0.05$ ], and a significant Context by Visual Field by Prime Type interaction $[F(1,2)=22.23, p<0.05]$. The nature of this interaction, as depicted in Figure 2, was further explored by analyzing the effects of Visual Field and Prime Type separately in the two context conditions. In the semantic context condition, decision latencies were affected only by Prime Type $[F(1,2)=$ $13.08, p<0.07$ ]. While marginal, this effect indicated 
ated condition. Response latencies for targets preceded by RVF primes did not differ as a function of orthographic similarity. Examination of the individual means confirmed that this pattern was present in each of the three neglect patients tested.

\section{Experiment 2b: Discrimination of Orthographically Similar Lexical Information}

The discrimination data from both testing sessions were collapsed so that the data analysis was conducted with a total of $\mathbf{8 0}$ trials per subject. To determine overall level of performance, percentage correct was calculated as a function of visual field and orthographic similarity. As Table 4 indicates, neglect patients performed at chance for LVF targets (chance calculated to lie between 37 and $63 \%$ ), regardless of whether they were orthographically similar or dissimilar. In contrast, they performed above chance for RVF targets, regardless of whether they were orthographically similar or dissimilar. Normal control subjects performed above chance in all conditions.

Percent correct discrimination was also analyzed in a $2 \times(2 \times 2)$ repeated measures ANOVA to determine if orthographic similarity affected either group's performance differently across left versus right visual field. Significant effects were found for Group $[F(1,11)=$ $79.80, p<0.001]$, Visual Field $[F(1,2)=21.36, p<0.05]$, and Orthographic Similarity $[F(1,11)=25.51, p<$ $0.001]$. The interaction between Visual Field and Group was also significant $[F(1,2)=32.731, p<0.001]$. Comparisons indicated that neglect patients performed significantly more accurately for targets presented in the RVF than for targets presented in the $\operatorname{LVF}[F(1,2)=$ $32.31, p<0.001$ ], whereas normal control subjects performed similarly regardiess of the visual field in which the target was presented $[F(1,2)<1]$. The three-way interaction was also significant $[F(1,2)=6.18, p<0.05]$. A series of means comparisons revealed that neglect patients' discrimination performance for LVF targets was more severely impaired when the foil was orthographi- cally similar compared to orthographically dissimilar $[F(1,2)=12.06, p<0.01]$. However, their performance did not exceed chance in either of these conditions.

The findings from the discrimination task again confirm that information that is processed sufficiently to activate semantic information is not specified sufficiently to influence discrimination performance. The absence of an effect of orthographic similarity even for targets initially presented in the RVF suggests that the double simultaneous displays consisting of the target word and filler XXXs were sufficient to prevent attention from being directed exclusively to the rightmost side of the RVF.

\section{GENERAL DISCUSSION}

The current study investigated the presence and nature of semantic priming from neglected lexical information in hemispatial neglect patients. Toward this end, two sets of experiments were conducted to determine (1) if orthographic information falling within the neglected visual field is specified sufficiently to activate abstract lexical and semantic representations, and (2) if observed priming effects are based on explicitly perceived orthographic information contained within the rightmost side of the prime word, or based on fully specified, implicitly processed orthographic and semantic representations.

The pattern of results from the first pair of experiments was clear. Even though patients could not explicitly identify words appearing in their neglected visual field, these words nevertheless influenced the speed with which subsequent lexical decisions were made. Importantly, the magnitude of the priming effects was equivalent in both visual fields and was similar to that found in normal control subjects. The basic semantic priming effect was replicated in three additional neglect patients in the semantic condition of Experiment $2 a$, where again, results from the discrimination task indicated that LVF primes were not processed to levels sufficient for visual awareness.

Table 4. Percentage Correct in Experiment 2b

\begin{tabular}{|c|c|c|c|c|}
\hline & \multicolumn{2}{|c|}{$\begin{array}{l}\text { Left visual field } \\
\text { Ortbograpbically }\end{array}$} & \multicolumn{2}{|c|}{$\begin{array}{l}\text { Rigbt visual field } \\
\text { Ortbograpbically }\end{array}$} \\
\hline & Similar & Dissimilar & Similar & Dissimilar \\
\hline Neglect 1 & 35 & 60 & 85 & 70 \\
\hline Neglect 2 & 40 & 55 & 100 & 95 \\
\hline Neglect 3 & 45 & 60 & 60 & 85 \\
\hline Neglect patients $(n=3)$ & 40 & 58 & 82 & 83 \\
\hline Control subjects $(n=10)$ & 96 & 97 & 91 & 96 \\
\hline
\end{tabular}


patients ranged in age from 64 to 76 years (mean age $71 \pm 4.74$ ); education ranged from 8 to 13 years (mean education $11 \pm 2.08$ ). The control subjects ranged in age from 59 to 73 years (mean age $65 \pm 4.45$ ); education ranged from 12 to 16 years (mean education $14 \pm 1.48$ ). Three patients and 10 normal control subjects participated in Experiments $2 \mathrm{a}$ and $2 \mathrm{~b}$. The patients ranged in age from 70 to 74 years (mean age $72 \pm 2.08$ ); education ranged from 13 to 14 years (mean education $13 \pm 0.58$ ). The control subjects ranged in age from 59 to 73 years (mean age $72 \pm 3.0$ ); education ranged from 12 to 16 years (mean education $14 \pm 2.0$ ). All subjects were righthanded. For the patients, CT or MRI confirmed a single unilateral right hemisphere infarction and neuropsychological testing revealed contralesional hemispatial neglect. Patients were excluded if there was evidence of a left hemisphere lesion or a history of substance abuse.

Hemispatial neglect was assessed with a number of tests including horizontal line bisection, figure drawing, word reading (mono- and bisyllabic), detection of double simultaneous stimulation for visual stimuli, and a series of target cancellation tasks (including letters, symbols, and lines). Patients who participated displayed evidence of LVF neglect on at least two of these assessments. Of note, however, is the fact that only one of the patients had mild neglect dyslexia. All patients had normal visual fields to single stimulation. Table 5 displays demographic, clinical, and lesion localization information for the patients included in this sample.

\section{Apparatus}

All experiments employed an Apple Macintosh microcomputer. The software (Psychlab) was designed for the control of stimulus presentation, timing, and the recording of responses with millisecond accuracy. Manual responses were recorded by two telegraph keys interfaced with the computer via a modified keyboard. Face position relative to the computer screen was maintained with a standard opthalmological chin rest centered 12 in. from the screen. The chin rest height was adjustable so that the individual subject's eyes were at the same level as the fixation point presented on the video monitor.

\section{General Procedure}

Each neglect patient was tested in a quiet, softly lit laboratory space located at the Braintree Rehabilitation Hospital, MA. Control subjects were tested at the Brockton/West Roxbury Department of Veterans Affairs Medical Center (DVAMC) and the Boston DVAMC. The entire protocol, including the experiments and neuropsychological testing (for the patients), was conducted over a varying number of sessions ranging from one-half to $1 \mathrm{hr}$ each, depending on the patient's ability to sustain attention. In the initial session(s), background informa- tion and neuropsychological data were collected. The two sets of experiments were administered in two subsequent sessions. Within each session, the priming task preceded the discrimination task. We felt this to be the most conservative order of testing because it would not permit exposure during the discrimination task to influence the presence/absence of priming during the priming task. All the patients completed the entire protocol within a 2-week period.

\section{Stimuli and Procedure}

\section{Experiment Ia}

The target stimuli were constructed from 120 high-frequency words chosen from the norms of Francis and Kucera (1982) and were 3 to 5 letters in length. Half of these stimuli were used as real word targets, and the remaining stimuli matched for word length, were changed into nonwords by substituting one letter, but respecting the rules of English orthography. The letter that was substituted occurred equally often on the left and on the right side of the word.

The priming stimuli were also constructed from 120 high-frequency words, 3 to 5 letters in length. For the 60 trials in which the target stimulus was a real word, three different types of primes were used. One-third (i.e., 20) of the primes was semantically related to the target, one-third was unrelated to the target, and the final onethird was composed of nonword filler stimuli. For the 60 trials in which the target stimulus was a nonword, half of the primes were real words, and the other half were nonwords.

All priming displays were composed of a priming stimulus and a neutral "filler" stimulus (string of Xs equal in length to the priming stimuli). The presentation of the prime and filler stimuli was counterbalanced across visual field so that half of the primes in each condition was presented in the LVF and half was presented in the RVF. The double simultaneous displays were intended to increase the likelihood that attention would be focused centrally. The order of the trials was randomized and remained constant across subjects.

The beginning of each trial was signaled with an asterisk centered on a video display monitor for $\mathbf{5 0 0}$ msec. The asterisk was followed immediately by the presentation of the prime and filler stimuli for $200 \mathrm{msec}$. The relatively short exposure of the priming stimuli was intended to minimize the possibility of saccadic eye movements. The prime and filler were centered $1.5^{\circ}$ to the left and right of the center of the display monitor. Following an interstimulus interval (ISI) of $400 \mathrm{msec}$, the target string was displayed centrally and remained on the screen until a response was made by the subject. Yes/No (i.e., word/nonword) decisions and accuracy were recorded automatically by the computer.

The subjects were told that two events would appear on the computer screen, one immediately following the 
fixation point, and to be ready for the presentation of two stimuli, which would be presented only briefly. They were then asked to attend to the two vertically aligned stimuli, and to depress one of two keys, also aligned vertically, to indicate which of the two stimuli appearing on the video monitor they had just viewed. They were asked to depress the telegraph key labeled "TOP" if they thought they had just seen the word located on the top and to depress the key labeled "BOTTOM" if they thought they had just seen the word located on the bottom.

After practice, subjects were presented with $40 \mathrm{ex}$ perimental trials, divided into 2 blocks of 20 trials each. During the course of testing, the patients were carefully watched by the examiner to ensure that they maintained attention to the task. Patients were given a brief rest after each block. All subjects were instructed to respond as accurately as possible.

\section{Experiment $2 a$}

In total this experiment was composed of 240 trials; half of the trials comprised the semantic priming condition and the other half comprised the orthographic priming condition. These conditions were blocked and administered to subjects on separate testing days.

The target stimuli in the semantic context condition were constructed from 120 high-frequency words chosen from the norms of Francis and Kucera (1982). Half of these stimuli was used as real word targets, and the remaining stimuli matched for word length were changed into nonwords by substituting one letter, but respecting the rules of English orthography.

The priming stimuli were also constructed from 120 high-frequency words. For the 60 trials in which the target stimulus was a real word, three different types of primes were used. One-third (i.e., 20) of the primes was semantically related to the target, one-third was unrelated to the target, and the final one-third was composed of nonword filler stimuli. For the 60 trials in which the target stimulus was a nonword, 40 of the primes were real words, and the remaining 20 were nonword primes.

For the orthographically mediated context condition, the first letter of each prime word in the semantic context condition was replaced to form orthographically similar but semantically unrelated primes that matched the original prime word with regard to word frequency and length. All other stimuli were identical to those in the semantic condition. Experiment $2 a$ was otherwise identical in procedure to Experiment 1a.

\section{Experiment $2 b$}

In total, this experiment consisted of 80 trials, split evenly across two testing sessions. Within each session, 40 words served as targets and 40 served as foils. All of the stimuli were words that served as primes in Experi- ment 2a. Each word was presented once during each testing session.

Within each session, a target word was presented in the left visual field on half of the trials, and in the right visual field on the remaining half of the trials. Following each presentation of these lateralized stimuli, a test trial was presented, consisting of the target word as well as a foil, presented vertically, one on top of the other. On half of the trials ( $n=10$ per session), foils were orthographically similar (with the exception of the left-most letter) but semantically unrelated to the target (e.g., corn and horn). On the other half of the trials, foils were semantically unrelated and orthographically dissimilar to the target. The positioning of the correct choice on either the top or bottom of the display was counterbalanced across trials.

Each subject received one list per testing session. A total of 4 lists were prepared that counterbalanced for orthographic similarity and visual field. That is, target items that were orthographically similar in one list were dissimilar in the second list. One list was administered following the semantic priming condition of Experiment 2a and one list was administered following the orthographic priming condition. Experiment $2 \mathrm{~b}$ was otherwise identical in procedure to Experiment $1 \mathrm{~b}$.

\section{Acknowledgments}

This work was supported by Grant NS29342-02 to William P. Milberg at the GRECC, Brockton/West Roxbury DVAMC, and Harvard Medical School. We express our thanks to Dr. John Ween for screening our patients. Thanks also to A. Joelle Grande and Elisa Bolton for testing subjects and to Patrick Kilduff for providing software programming and computer support.

Reprint request should be sent to Regina McGlinchey-Berroth, GRECC/VA Medical Center (182), 1400 VFW Parkway, West Roxbury, MA 02132.

\section{REFERENCES}

Audet, T., Bub, D., \& Lecours, A. R. (1991). Visual neglect and left-sided context effects. Brain and Cognition, 16, 11-28.

Berti, A., \& Rizzolatti, G. (1992). Visual processing without awareness: Evidence from unilateral neglect. Journal of Cognitive Neuroscience, 4, 345-351.

Cheesman, J., \& Merikle, P. M. (1986). Distinguishing conscious from unconscious perceptual processes. Canadian Journal of Psycbology, 40, 343-367.

Dagenbach, D., Carr, T. H., \& Barnhardt, T. H. (1990). Inhibitory semantic priming of lexical decisions due to failure to retrieve weakly activated codes. Journal of Experimental Psychology, 16, 328-340.

Farah, M. J., Monheit, M. A., \& Wallace, M. A. (1991). Unconscious perception of "extinguished" visual stimuli: Reassessing the evidence. Neuropsycbologia, 29, 949-958.

Forster, K. I. (1987). Form-priming with masked primes: The best-match hypothesis. In M. Coltheart (Ed.), Attention and performance (pp. 127-146). Hillsdale, NJ: Erlbaum.

Forster, K. I., Booker, J., Schacter, D. L., \& Davis, C. (1990). Masked repetition priming: Lexical activation or novel 
Errata: In Semantic Processing and Orthograpbic Specificty in Hemispatial Neglect, by McGlinchey-Berroth et al. (Journal of Cognitive Neuroscience 8(3): 291-304), Figures 1 and 2 were incorrect. The correct figures appear below.

In addition, the first sentence of the abstract for that article should have read, "Two sets of experiments, each consisting of a semantic priming task and a discrimination task, investigated the processing of lexical information in the neglected visual field."

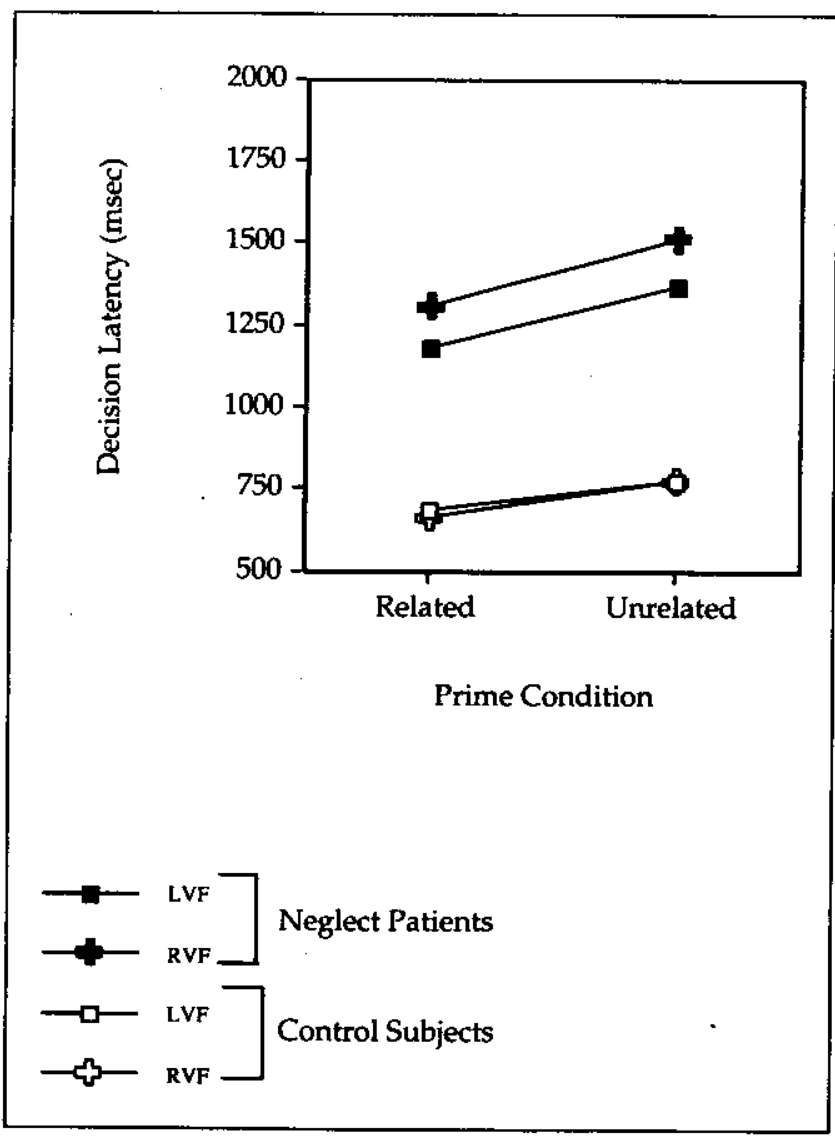

Figure 1. Mean lexical decision latencies as a function of priming condition and visual field in Experiment 1a for neglect patients and normal control subjects.

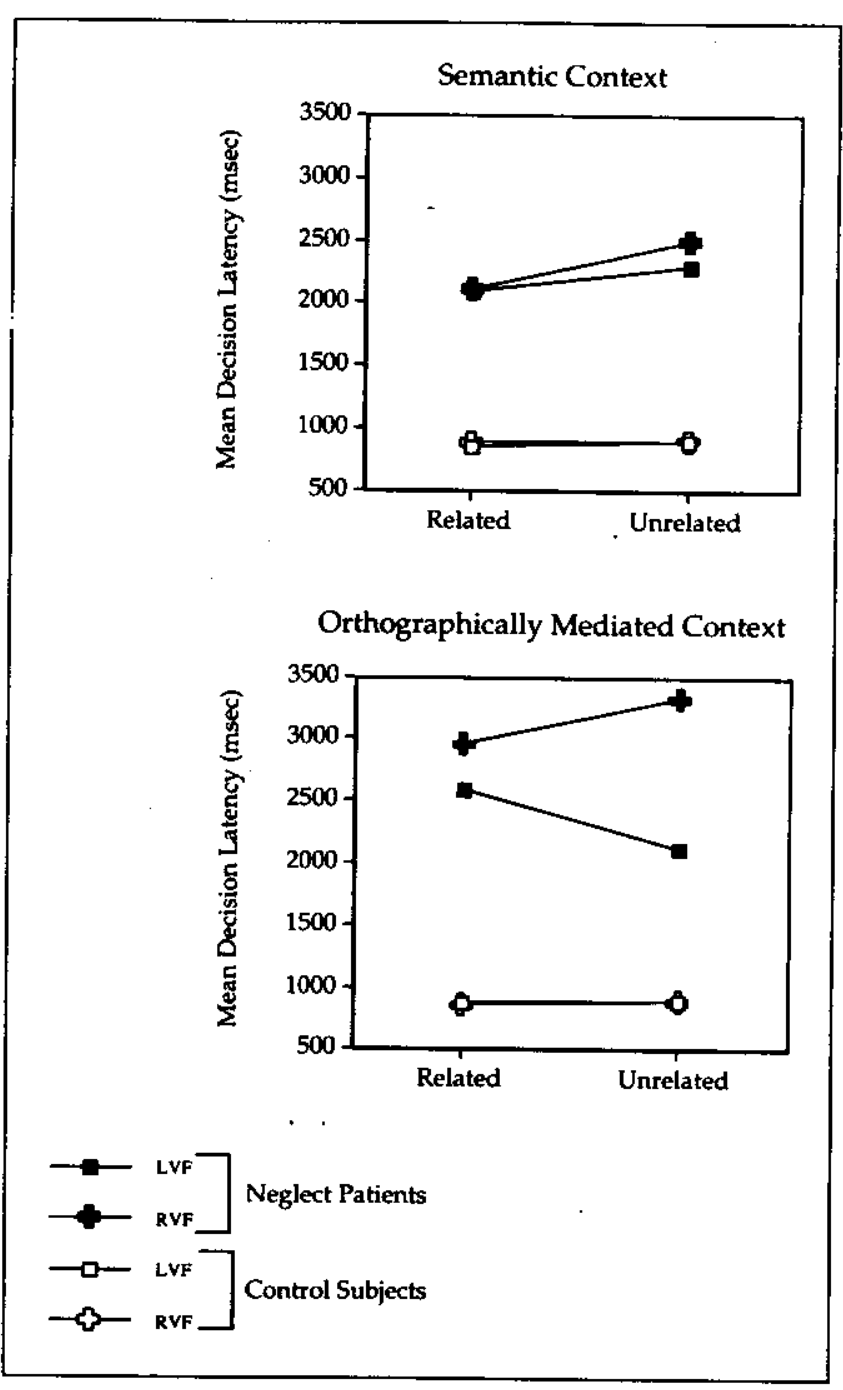

Figure 2. Mean lexical decision latencies as a function of context condition, priming condition, and visual field in Experiment 2a for neglect patients and normal control subjects. 